\title{
Thymectomy for paraneoplastic stiff-person syndrome associated with invasive thymoma
}

\author{
Takashi Iwata, MD, ${ }^{a}$ Kiyotoshi Inoue, MD, ${ }^{a}$ Shinjiro Mizuguchi, MD, ${ }^{a}$ \\ Ryuhei Morita, MD, ${ }^{a}$ Takuma Tsukioka, MD, ${ }^{a}$ and Shigefumi Suehiro, MD, ${ }^{\mathrm{b}}$ Osaka, Japan
}

tiff-person syndrome (SPS) is a rare autoimmune neurologic disorder characterized by painful tonic spasms in systemic skeletal muscles. ${ }^{1}$ Anti-glutaminic acid decarboxylase (GAD) antibodies are produced with disturbing synthesis of $\gamma$-aminobutylic acid, one of the important suppressive neurotransmitters in the central nervous system. In some patients SPS is reportedly accompanied by thymomas, ${ }^{2-4}$ and in these patients surgical resection of the malignancy reportedly reduces the neurologic symptoms of SPS. We report herein the clinical course of a patient with SPS associated with invasive thymoma.

\section{Clinical Summary}

A 79-year-old woman presented with severe, painful tonic spasms of her left leg as a result of a fall. Conservative therapy by an orthopedic surgeon subdued the leg pain for 2 weeks; however, stiffness remained. After this, her voice began to become unclear, and she complained of dysarthria. She lost $10 \mathrm{~kg}$ in weight in 3 months. She was therefore referred to a neurologist in our hospital. She was not mentally disturbed. Her muscles were generally weakened, but her reflexes were normal. She complained of severe sharp pain in her left knee with a fine touch and cramping pain without touching.

Chemical studies revealed that she was not diabetic. Her serum titer of anti-GAD antibody was 56,400 U/mL. An electromyogram demonstrated continuous discharge at rest in her left leg, which disappeared immediately with intravenous administration of diazepam. The left leg stiffness also disappeared after injection of diazepam. Chest computed tomography showed a round mass enhanced mildly in the anterior mediastinum (Figure 1). She was given a diagnosis of paraneoplastic SPS associated with thymoma and transferred to our department for surgical treatment.

She underwent intravenous hyperalimentation therapy and was treated initially with oral administration of diazepam, dantrolene, tizanidine, and baclofen, respectively. However, no drug had an effect, and the symptoms continued to exacerbate. Steroid pulse therapy was therefore attempted but again did not improve her

From the Departments of Thoracic Surgery ${ }^{\mathrm{a}}$ and Cardiovascular Surgery, ${ }^{\mathrm{b}}$ Osaka City University Hospital, Osaka, Japan

Supported by Osaka City University Medical School.

Address for reprints: Takashi Iwata, MD, Department of Thoracic Surgery, Osaka City University Hospital, 1-4-3 Asahi-machi, Abeno-ku, Osaka, 545-8585 Japan (E-mail: taiwata@med.osaka-cu.ac.jp).

J Thorac Cardiovasc Surg 2006;132:196-7

$0022-5223 / \$ 32.00$

Copyright $\odot 2006$ by The American Association for Thoracic Surgery doi:10.1016/j.jtcvs.2006.03.030 symptoms. Finally, intravenous immunoglobulin (IVIG) therapy was initiated. Three hours after the first administration, the painful stiffness of the left leg dramatically improved, and she was able to bend her left knee without pain. She started eating on day 2 after initiating IVIG, without dysphagia. One week later, her general status, including pulmonary function, showed overall improvement.

After the above, extended thymectomy was carried out after achievement of total intravenous anesthesia with propofol; muscle relaxants were not used. A well-encapsulated thymic tumor was removed with all the thymic tissue. The tumor was round in shape and approximately $5 \mathrm{~cm}$ in diameter and histologically diagnosed as an invasive thymoma of mixed type (Figure 2). On the fourth postoperative day, the patient was able to start walking. Her serum anti-GAD antibody titer reduced to $11,600 \mathrm{U} / \mathrm{mL} 1$ week after the operation, and her symptoms dramatically improved without the need for medication. Two weeks after the operation, her titer increased to $22,500 \mathrm{U} / \mathrm{mL}$, without any relapsing symptoms. After IVIG was performed twice to prevent relapse, she was discharged asymptomatic without medication.

Three weeks later, she experienced a repeat fall in which she hit her head on the floor. After a few hours, she experienced systemic painful stiffness and convulsions of both legs. She was admitted with relapsing SPS. IVIG was performed immediately but was ineffective. Plasmapheresis was therefore carried out 3 times, improving her muscular stiffness gradually. Baclofen and clonidine were started, and she eventually became asymptomatic. Twelve months after the operation, the patient is doing well, without obvious evidence of relapse or exacerbated SPS.

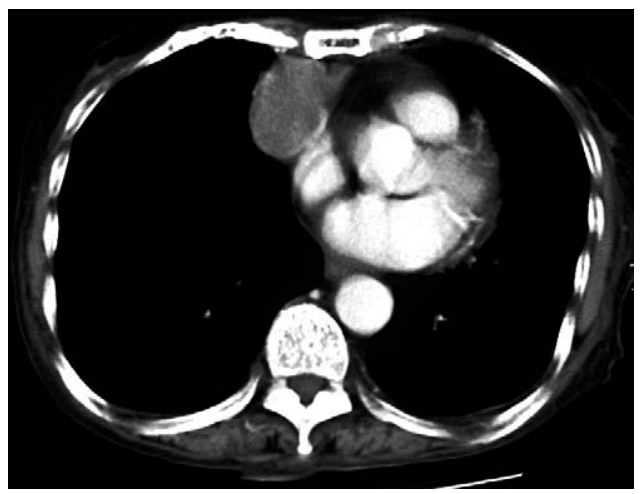

Figure 1. Chest computed tomography demonstrated a round, slightly enhanced thymoma $5 \mathrm{~cm}$ in diameter in the anterior mediastinum. 


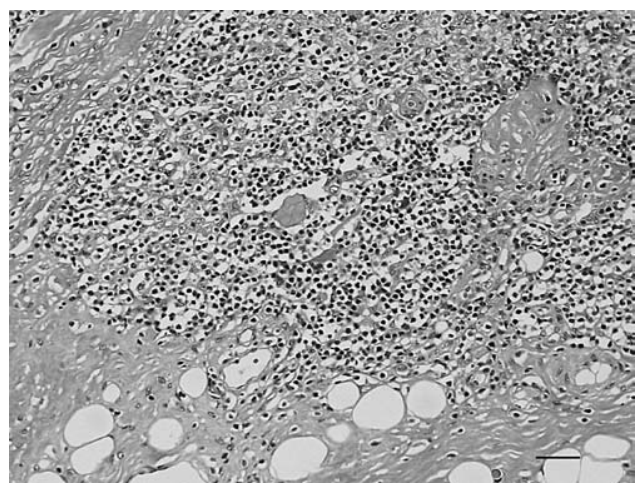

Figure 2. Pathologic findings of the resected thymoma. (Original magnification $200 \times$.) Intracapsular invasion and both small lymphocytic and epithelial components were observed. The patient was given a diagnosis of an invasive thymoma of mixed type.

\section{Discussion}

In patients with paraneoplastic SPS, surgical removal of the associating neoplasm is thought to be important in reducing neurologic symptoms. Sommer and colleagues ${ }^{5}$ previously reported that rats experienced spastic stiffness like human SPS with injection of purified IgG taken from a patient with paraneoplastic SPS associated with breast cancer. This suggests that paraneoplastic production of autoantibodies could be associated with paraneoplastic SPS.

Clinically, Hagiwara and associates ${ }^{4}$ and Tanaka and cowork$\mathrm{ers}^{3}$ reported SPS cases in which the painful stiffness was reduced by surgical resection of a thymoma. After resection, serum autoantibody titers should be reduced in such cases; however, in our patient the GAD antibody titer was once again increased 2 weeks after resection, despite a lack of symptoms. Moreover, as a result of traumatic stimulation, SPS suddenly relapsed in the clinically asymptomatic period after surgical intervention. This clinical course suggests that surgical removal of the tumor does not always mean the prevention of autoantibody productivity and is not always a curative treatment for paraneoplastic SPS, as with myasthenia gravis associated with thymoma.

\section{References}

1. Murinson BB. Stiff-person syndrome. Neurologist. 2004;10:131-7.

2. Nicholas AP, Chatterjee A, Arnold MM, Claussen GC, Zorn GL Jr, Oh SJ. Stiff-persons' syndrome associated with thymoma and subsequent myasthenia gravis. Muscle Nerve. 1997;20:493-8.

3. Tanaka $\mathrm{H}$, Matsumura A, Okumura $\mathrm{M}$, Kitaguchi M, Yamamoto S, Iuchi K. Stiff man syndrome with thymoma. Ann Thorac Surg. 2005;80:739-41.

4. Hagiwara H, Enomoto-Nakatani S, Sakai K, Ugawa Y, Kusunoki S, Kanazawa I. Stiff-person syndrome associated with invasive thymoma: a case report. J Neurol Sci. 2001;193:59-62.

5. Sommer C, Weishaupt A, Brinkhoff J, Biko L, Wessig C, Gold R, et al. Paraneoplastic stiff-person syndrome: passive transfer to rats by means of IgG antibodies to amphiphysin. Lancet. 2005;365:1406-11.

\title{
Postoperative pulmonary embolism detected with multislice computed tomography in lung surgery for cancer
}

\author{
Giuliano Daddi, MD, ${ }^{a}$ Giulia Milillo, MD, ${ }^{\mathrm{b}}$ Luciano Lupattelli, MD, ${ }^{\mathrm{c}}$ Mark Ragusa, MD, ${ }^{a}$ Angelo Lemmi, MD, \\ Francesco Puma, MD, ${ }^{d}$ and Giancarlo Agnelli, ${ }^{b}$ for the Pulmonary Embolism in Thoracic Surgery Study Group, Perugia, Italy
}

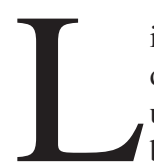
imited information is currently available on the incidence of venous thromboembolism (VTE) in patients undergoing lung surgery for cancer. ${ }^{1,2}$ Pulmonary embolism (PE) has been found to be a frequently fatal postoperative complication, as confirmed by autopsy in $15.2 \%$ of

From the Thoracic Surgery Unit, ${ }^{a}$ and Departments of Internal Medicine and Vascular Diseases ${ }^{\mathrm{b}}$ and Radiology, ${ }^{\mathrm{c}}$ Perugia University, Perugia, Italy; and Thoracic Surgery Unit, Perugia University at Terni, ${ }^{\mathrm{d}}$ Italy.

Presented as a poster at "Chest 2004," Annual Meeting of the American College of Chest Physicians, Seattle, Wash, October 23-28, 2004.

Address for reprints: Mark Ragusa, MD, S.C. Chirurgia Toracica, Università di Perugia, Ospedale Silvestrini, Via G. Dottori, 06156, Perugia, Italy (E-mail: drfastnet@bigfoot.com).

J Thorac Cardiovasc Surg 2006;132:197-8

$0022-5223 / \$ 32.00$

Copyright $\odot 2006$ by The American Association for Thoracic Surgery doi:10.1016/j.jtcvs.2006.03.036 postresectional deaths. ${ }^{3}$ The clinical burden of PE in surgical patients is underestimated, probably because the majority of VTE remains asymptomatic or associated with aspecific symptoms. Several factors increase the thromboembolic risk in patients undergoing lung cancer surgery: intrinsic procoagulant effect of cancer, extensive surgical intervention, dependent limb position in the operating room, and vessel injury consequent to the operation.

$\mathrm{PE}$ in patients undergoing thoracic surgery has some peculiarities. Indeed, in thoracic surgery, in addition to the established risk factors for VTE, local factors related to surgical technique, direct vascular injury, or both could play a remarkable role. ${ }^{4}$

The aim of this study was to assess the incidence of PE after lung surgery for cancer by using multislice computed tomographic (MSCT) scanning.

\section{Methods}

The Pulmonary Embolism in Thoracic Surgery Study was a prospective study performed to identify the incidence of PE by means of MSCT scanning after lung surgery for cancer. 